\title{
Efficacy of multimodal treatment for leptomeningeal metastases in a lung cancer harboring an EGFR mutation
}

\author{
Daisuke Morichika' \\ Toshio Kubo² \\ Hiroko Gotoda' \\ Tomoki Tamura' \\ Kadoaki Ohashi' \\ Katsuyuki Hotta' \\ Masahiro Tabata ${ }^{2}$ \\ Kazuhiko Kurozumi ${ }^{3}$ \\ Mitsune Tanimoto ${ }^{4}$ \\ Katsuyuki Kiura' \\ 'Department of Respiratory \\ Medicine, ${ }^{2}$ Center for Clinical \\ Oncology, Okayama University \\ Hospital, Okayama, Japan; \\ ${ }^{3}$ Department of Neurological \\ Surgery, ${ }^{4}$ Department of Hematology, \\ Oncology, and Respiratory Medicine, \\ Okayama University Graduate \\ School of Medicine, Dentistry and \\ Pharmaceutical Sciences, Okayama \\ University, Okayama, Japan
}

This article was published in the following Dove Press journal:

OncoTargets and Therapy

22 March 2016

Number of times this article has been viewed

\begin{abstract}
For lung cancer patients with epidermal growth factor receptor (EGFR) mutations, the advent of EGFR tyrosine kinase inhibitors (TKIs) has prolonged survival rates. Even though disease sites have been well controlled by EGFR-TKIs, some patients develop carcinomatous meningitis, which reduces their quality of life drastically. Although multidisciplinary approaches have improved patient survival and quality of life, the outcomes are not yet satisfactory. We report the case of a 54-year-old Japanese woman diagnosed with leptomeningeal metastases (LM) from a lung adenocarcinoma harboring an EGFR exon 21 L858R point mutation. She was treated with gefitinib for 2 months, and symptoms of LM emerged during the treatment period. Although the treatment was switched to erlotinib, disturbance of consciousness worsened because of progressive hydrocephalus. Because all extracranial lesions remained responsive to treatment, and the exon $20 \mathrm{~T} 790 \mathrm{M}$ point mutation was not detected in cerebrospinal fluid, we placed a ventriculoperitoneal shunt. The patient's disturbed consciousness improved dramatically after the shunt was placed; however, the optic and auditory nerve impairments due to direct invasion of LM lesions into nerve canals persisted. Administration of bevacizumab subsequent to whole-brain radiotherapy reduced the cranial nerve impairment, and the patient survived for 10 months. In conclusion, a combination of erlotinib and ventriculoperitoneal shunt was effective for hydrocephalus, and the immediate administration of additional therapies, including
\end{abstract} bevacizumab and radiation therapy, was useful in a patient suffering from LM.

Keywords: bevacizumab, erlotinib, ventriculoperitoneal shunt, leptomeningeal metastases, lung cancer, EGFR

\section{Introduction}

Leptomeningeal metastases (LM) are a relatively rare but fatal complication. The quality of life (QOL) of patients with LM is reduced significantly. The incidences of LM reported for solid cancers and for non-small-cell lung cancer (NSCLC) are 5\% and $1 \%$, respectively. ${ }^{1}$ Recently, the frequency of LM in NSCLC patients harboring epidermal growth factor receptor (EGFR) mutations has increased, because the use of EGFR tyrosine kinase inhibitors (TKIs) has prolonged patient survival. ${ }^{2}$ However, the efficacies of standard treatments for LM, including systemic chemotherapy, intrathecal chemotherapy, and whole-brain radiotherapy (WBRT), has room for improvement. ${ }^{2-4}$ In patients with LM due to NSCLC harboring EGFR mutations, the survival prognoses average 5.3 months, even with EGFR-TKI treatment, ${ }^{5}$ and this is not satisfactory. We report here the case of a patient with LM who with multimodal treatment (including EGFR-TKIs, ventriculoperitoneal [VP] shunt placement, WBRT, and bevacizumab) recovered substantial QOL and survived 10 months after her initial diagnosis.
Center for Clinical Oncology, Okayama

University Hospital, 2-5-I Shikata-cho,

Kita-ku, Okayama 700-8558, Japan

Tel +81862357227

Fax +81862328226

Email t-kubo@cc.okayama-u.ac.jp (c) (7) (1) 2016 Morichika et al. This work is published and licensed by Dove Medical Press Limited. The full terms of this license are available at https://www.dovepress.com/terms.php hereby accept the Terms. Non-commercial uses of the work are permitted without any further permission from Dove Medical Press Limited, provided the work is properly attributed. For permission for commercial use of this work, please see paragraphs 4.2 and 5 of our Terms (https://www.dovepress.com/terms.php).
her 

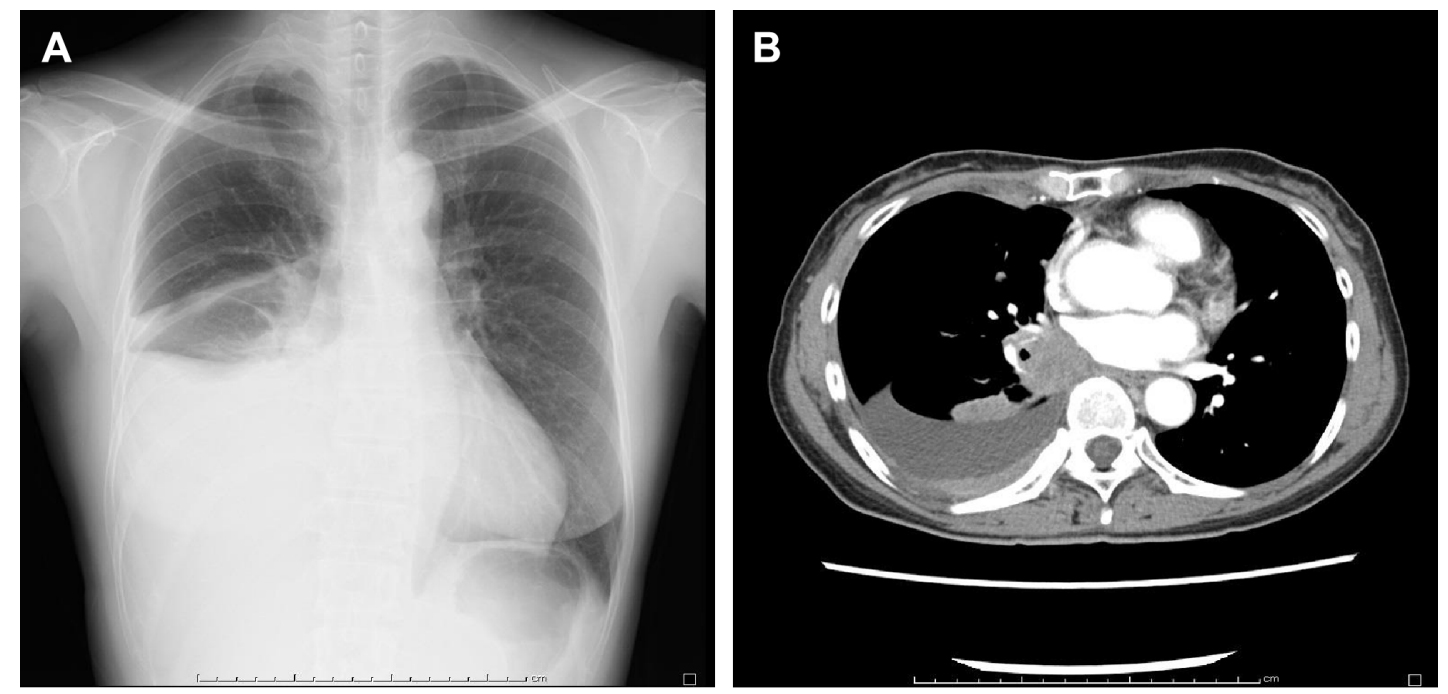

Figure I Radiological findings observed on initial visit.

Notes: (A) Chest radiograph revealed right pleural effusion. (B) Chest computed tomography revealed a mass in segment 6 of the right lower lobe.

\section{Case}

A 54-year-old Japanese woman was diagnosed with lung adenocarcinoma harboring an EGFR exon 21 L858R point mutation. Her clinical stage was T2aN0M1b, stage IV with multiple bone metastases and pleural dissemination (Figure 1). Gadolinium-enhanced MRI of the head demonstrated no specific findings.

Okayama university Institutional Review board does not require institutional review for case reports. This patient's family gave written informed consent to publish this report, and the identity of the patient has been protected.

The patient was treated with gefitinib $(250 \mathrm{mg} / \mathrm{d})$, and her primary tumor shrunk $80 \%$ in its longest diameter (a response evaluation criterion for solid tumors); her pleural effusion was slightly reduced. No unexpected adverse events were observed. Approximately 2 months after the initiation of gefitinib, a gait disorder and impaired cognition were noted, and the patient's performance status (PS) decreased from 1 to 3. Gadolinium-enhanced MRI of the head showed hydrocephalus without parenchymal metastases (Figure 2). Tests on cerebrospinal fluid (CSF) demonstrated elevated opening pressure $\left(270 \mathrm{mmH}_{2} \mathrm{O}\right)$, and cytology revealed adenocarcinoma cells. The patient was switched to erlotinib $(150 \mathrm{mg} / \mathrm{d})$ from gefitinib, and her symptoms were improved temporarily. The patient's pleural effusion disappeared without significant adverse events other than a grade 1 skin rash. Her symptoms were recrudescent after 3 months. A computed tomography (CT) scan of the head demonstrated that the hydrocephalus had worsened (Figure 3A). Its rapid progression left the patient in a deep coma, with a Glasgow Coma Scale score of 3 , and her life in danger.
Since all extracranial lesions remained responsive, and the exon $20 \mathrm{~T} 790 \mathrm{M}$ point mutation was not detected in cerebrospinal fluid, we determined that ventricular drainage and subsequent placement of a VP shunt might be an effective treatment option for the patient. Emergency ventricular drainage and subsequent placement of a VP shunt were performed. The patient recovered without disturbed or impaired cognition. Her PS improved from 4 to 2 , and she regained the ability to self-feed and to walk

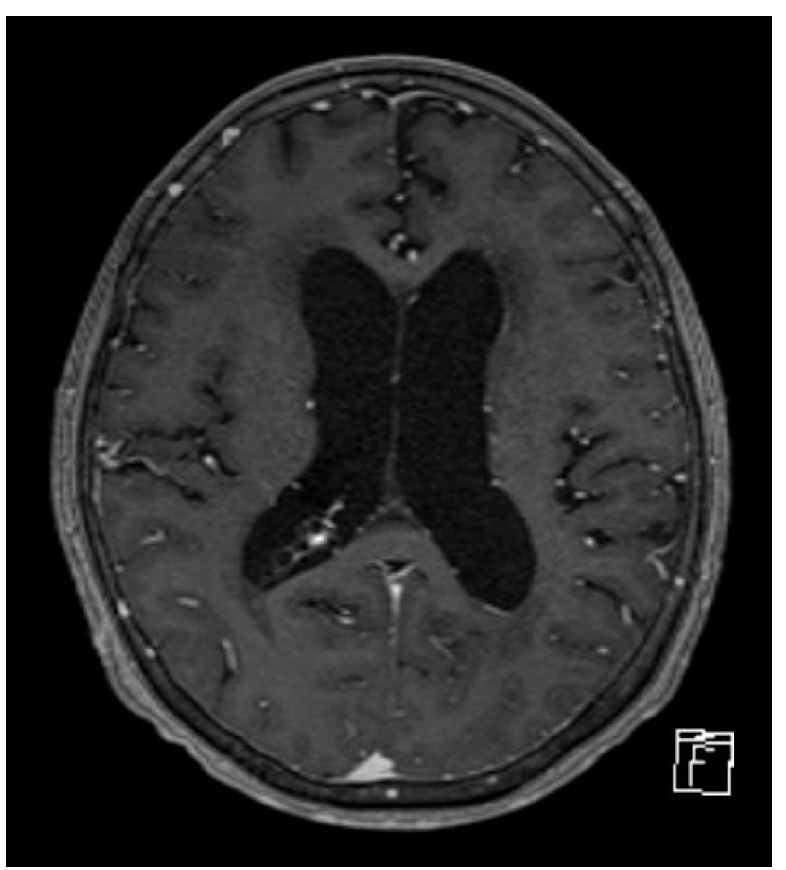

Figure 2 Gadolinium-enhanced MRI of the head on day 48 after the initiation of gefitinib.

Note: Hydrocephalus was detected without intracranial metastases. 

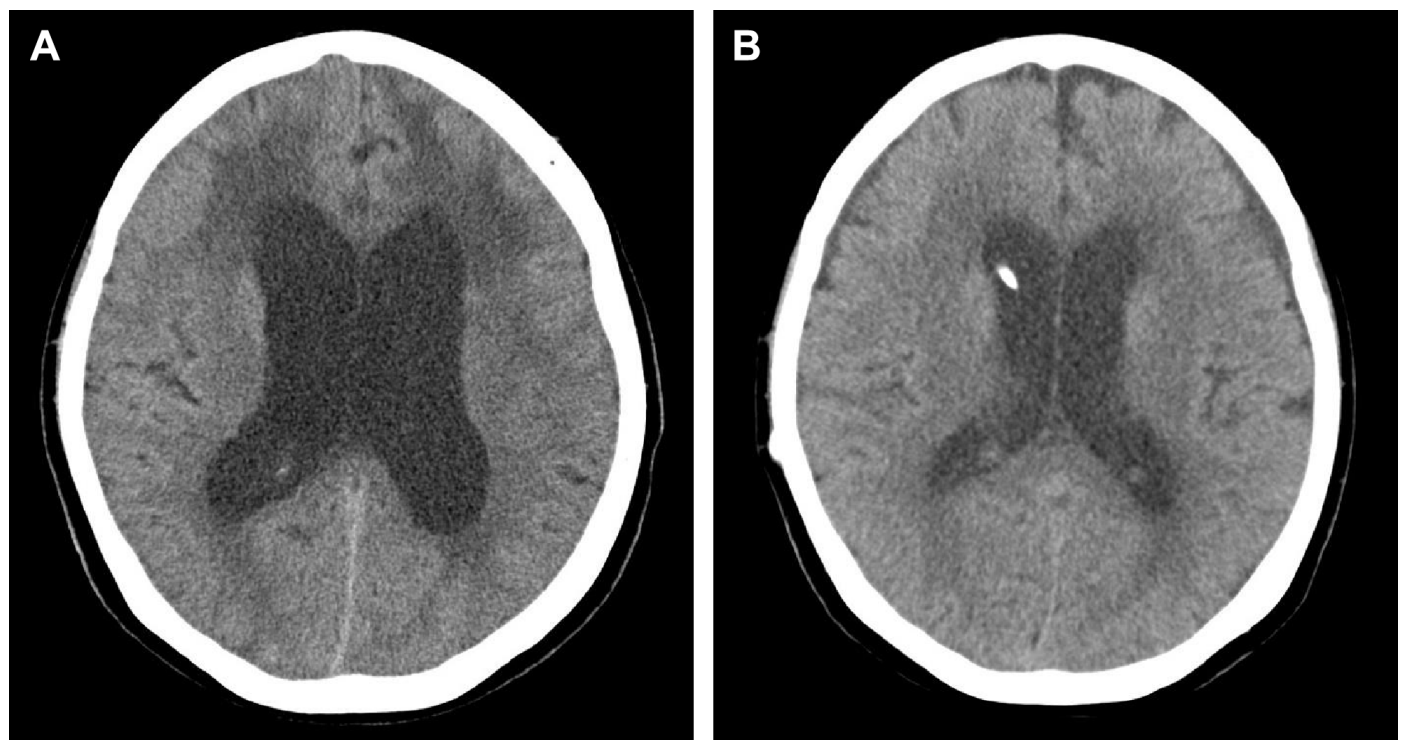

Figure 3 Radiological findings before and after VP shunt placement.

Notes: (A) Hydrocephalus on the head CT had worsened compared with the head MRI performed before the initiation of erlotinib. A low-density area was observed in the white matter of the bilateral frontal lobe because of intracranial hypertension 3 months after switching from gefitinib to erlotinib. (B) A head CT after VP shunt placement indicated that the malignant hydrocephalus had improved.

Abbreviations: VP shunt, ventriculoperitoneal shunt; CT, computed tomography.

by herself. Although this treatment approach improved her hydrocephalus (Figure 3B), complete blindness and a bilateral hearing disorder developed as a result of direct invasion of LM lesions into the relevant nerves. Gadoliniumenhanced MRI exhibited diffuse enhancement of the cranial dura mater. The optic canal and internal auditory canal were also enhanced (Figure 4). Although the patient continued erlotinib treatment and WBRT was performed concurrently ( 2 Gy single and daily fractions for 5 consecutive days each week with a total dose of $30 \mathrm{~Gy}$ ), the auditory disorder progressed slowly. After WBRT, the patient received systemic administration of bevacizumab (15 $\mathrm{mg} / \mathrm{kg}$ every 3 weeks $)$ in addition to erlotinib $(150 \mathrm{mg} / \mathrm{d})$. Three cycles of bevacizumab improved the patient's auditory disorder slightly and
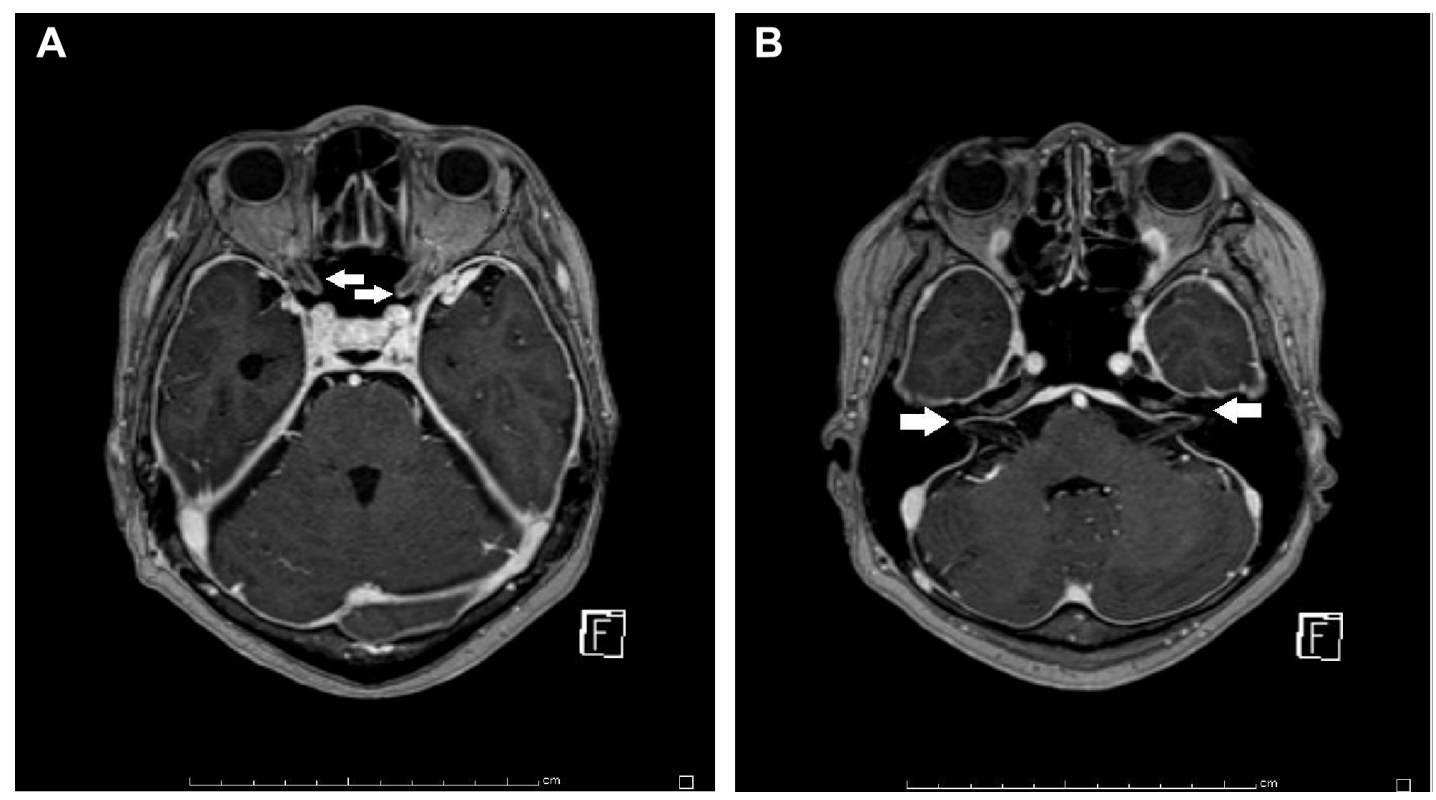

Figure 4 Gadolinium-enhanced MRI of the head after emergency placement of the VP shunt.

Notes: Gadolinium-enhanced MRI showed diffuse enhancement of the cranial dura mater. No evidence of brain parenchymal metastases was observed. (A) Thickening and enhancement of the dura mater in the optic nerve canal were observed (white arrows). (B) Thickening and enhancement of the dura mater in the auditory nerve canal were observed (white arrows).

Abbreviation: VP shunt, ventriculoperitoneal shunt. 

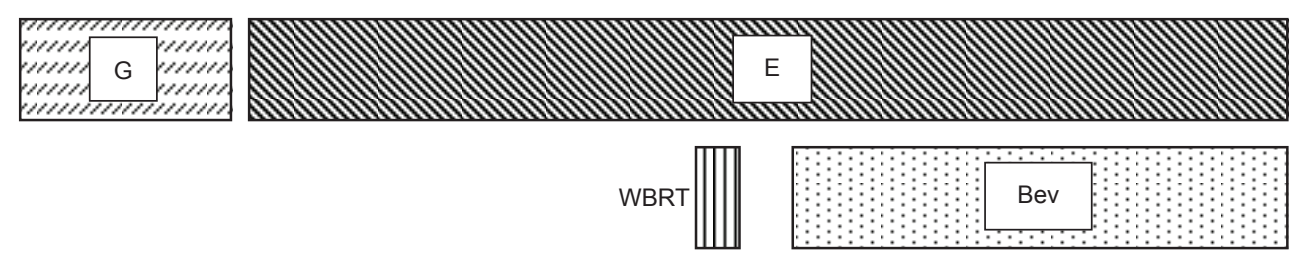

VP shunt
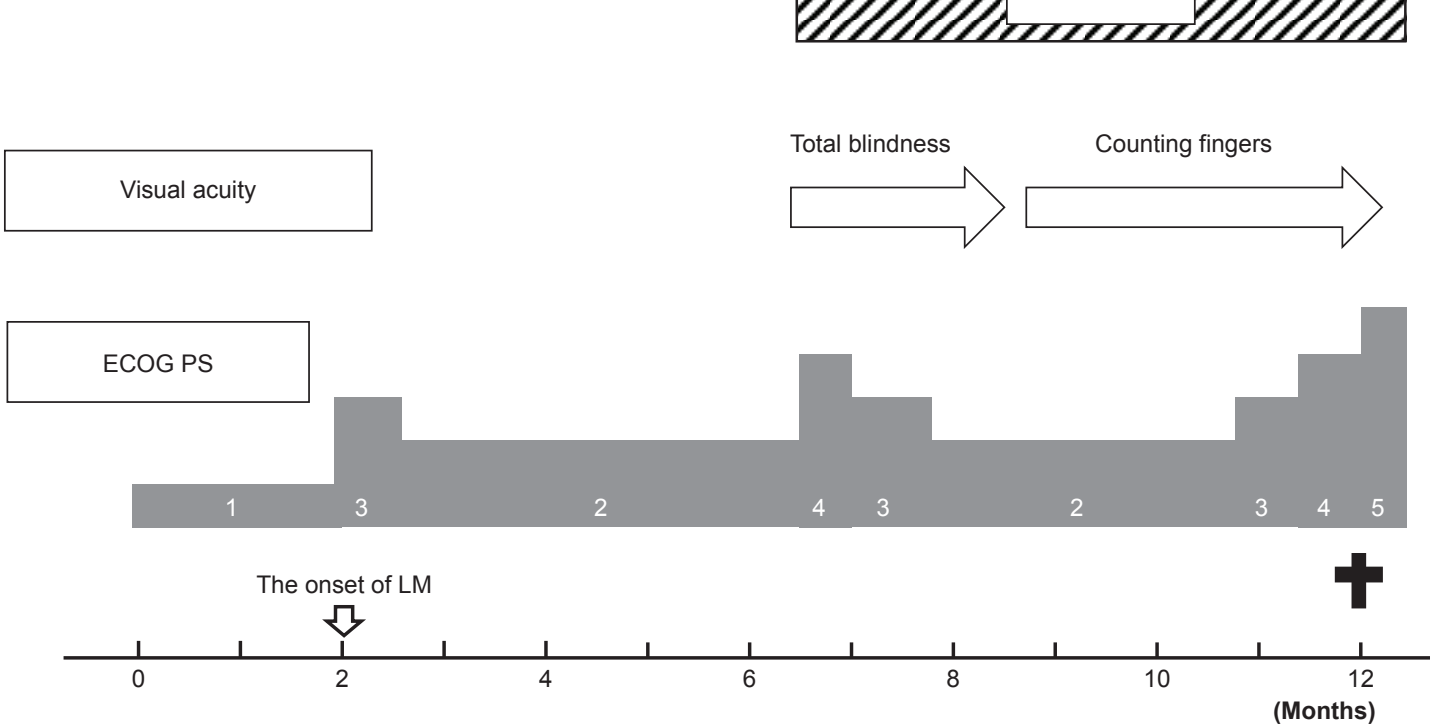

Figure 5 The clinical course of this patient.

Abbreviations: G, gefitinib; E, erlotinib; Bev, bevacizumab; WBRT, whole-brain radiotherapy; VP shunt, ventriculoperitoneal shunt; ECOG PS, Eastern Cooperative Oncology Group Performance Status; LM, leptomeningeal metastases.

improved her vision impairment from complete blindness to counting fingers. She continued bevacizumab for up to seven cycles without the development of carcinomatous peritonitis, and there were no severe toxicities noted, other than the grade 1 skin rash.

The patient's cranial nerve palsy progressed gradually. She died as a result of LM progression 10 months after the initial diagnosis (Figure 5).

\section{Discussion}

Recently, several studies have reported combination therapy with EGFR-TKIs and bevacizumab for NSCLC patients (Table 1).${ }^{6,7}$ However, to the best of our knowledge, this is the first case report of combination treatment with EGFRTKI and bevacizumab used for LM due to NSCLC. LM is the dissemination of malignant tumor cells through the CSF space. The infiltration of these spreading tumor cells to arachnoid villi and cranial nerves leads to hydrocephalus, cranial nerve palsy, and deterioration of the PS. Progression of hydrocephalus is often fatal. Recently, several studies have reported that EGFR-TKIs, especially erlotinib compared with gefitinib, might be effective treatments for LM in NSCLC with EGFR mutations. ${ }^{5,8-10}$ However, in some cases (including that of the patient presented here), the efficacy of EGFR-TKI was not sustained because the intracranial disease could not be controlled sufficiently. One reason for this is that, when used at a standard dose,

Table I The review of literature summarizing the clinical data on the combination of EGFR-TKIs and bevacizumab for non-smallcell lung cancer harboring an EGFR mutation

\begin{tabular}{|c|c|c|c|}
\hline & \multicolumn{2}{|l|}{ JO025567 } & \multirow{2}{*}{$\frac{\text { OLCSG I00 I }}{\text { Gefitinib + Bev }}$} \\
\hline & Erlotinib & Erlotiniv + Bev & \\
\hline \multicolumn{4}{|l|}{ EGFR mutation type } \\
\hline Ex 19 del (\%) & 52 & 53 & 57 \\
\hline L858R (\%) & 48 & 47 & 38 \\
\hline Others (\%) & 0 & 5 & 0 \\
\hline ORR (\%) & 63 & 69 & 74 \\
\hline Median PFS (months) & 10 & 16 & 14 \\
\hline \multicolumn{4}{|l|}{$\mathrm{AE}$} \\
\hline Grade 3-4 (\%) AEs & 51 & 91 & 60 \\
\hline Rash (\%) & 19 & 25 & 15 \\
\hline Diarrhea (\%) & 1 & I & \\
\hline Hypertension (\%) & 10 & 60 & 17 \\
\hline Proteinuria (\%) & 8 & 0 & 7 \\
\hline Hemorrhagic event (\%) & 0 & 3 & 2 \\
\hline
\end{tabular}

Note: Data from references. ${ }^{6}, 7$

Abbreviations: EGFR, epidermal growth factor receptor; TKI, tyrosine kinase inhibitor; Bev, bevacizumab; Ex, exon; del, deletion; ORR, objective response rate; $\mathrm{PFS}$, progression-free survival; $\mathrm{AE}$, adverse event; OS, overall survival. 
the concentration of EGFR-TKIs in CSF is unable to reach therapeutic levels. ${ }^{9,11}$ Togashi et al ${ }^{12}$ reported that the penetration rates of gefitinib and erlotinib were $1.13 \% \pm 0.36 \%$ and $2.77 \% \pm 0.45 \%$, respectively. A VP shunt has been reported to be an effective and palliative treatment for malignant hydrocephalus. ${ }^{13,14}$ In addition, the combination of a VP shunt and EGFR-TKIs, to maintain an adequate antitumor effect against malignant cells, could reduce fatalities caused by hydrocephalus, without leading to the development of carcinomatous peritonitis. ${ }^{15-17}$ Although this combination therapy was effective for improving symptoms caused by hydrocephalus, cranial nerve palsy caused by direct invasion of LM was not controlled. Therefore, additional treatment remained necessary in this situation.

Bevacizumab may be an attractive option for obtaining adequate tumor regression. Several studies have reported that vascular endothelial growth factor (VEGF) levels in the CSF of patients with LM were at least 14-fold higher than those seen in patients with other neurological disorders. Furthermore, elevated VEGF in the CSF was a strong predictor of poor survival. ${ }^{18,19}$ This evidence suggests that the VEGF signal can affect the development and progression of LM. Seto et $\mathrm{al}^{7}$ reported that erlotinib in combination with bevacizumab significantly prolonged the survival of patients with NSCLC harboring EGFR mutations. In addition, our group reported ${ }^{6}$ that gefitinib in combination with bevacizumab is an effective treatment for these NSCLC patients. Moreover, a Phase II prospective study indicated encouraging efficacy of bevacizumab for NSCLC with asymptomatic brain metastases. In that study, the response rate to bevacizumab with chemotherapy for brain metastases was $61.2 \%$, with a median duration of 8.1 months..$^{20}$ One of the reasons suggested for this efficacy is that antiangiogenic agents increase the intratumoral concentration of antitumor drugs. ${ }^{21-23}$

Although there is no evidence that bevacizumab can enter the CSF, these results suggest that even with the blood-brain barrier, bevacizumab can be effective against tumor cells that have invaded the brain parenchyma or dura mater in patients with LM. On the other hand, bevacizumab might not be as effective against the floating tumor cells found in CSF. In our strategy, the VP shunt discharged these tumor cells into the peritoneal cavity, where most chemotherapeutic agents can be delivered.

In addition, for this case, the mechanism by which bevacizumab exerted additional effects might be through a reduction in cerebral edema secondary to WBRT. ${ }^{24,25}$ A pilot study evaluating the efficacy of systemically administered bevacizumab in patients with neoplastic meningitis is ongoing (NCT00924820), and we expect this study to address whether bevacizumab is an effective treatment for LM.

In conclusion, the combination of erlotinib and a VP shunt was effective for hydrocephalus, and a multimodal treatment, including WBRT and bevacizumab, improved survival and QOL in a case of LM resulting from lung cancer harboring an EGFR mutation. The most important strategy in preventing LM-related cranial nerve palsy is the administration of adequate additional antitumor treatment as soon as symptoms occur after placement of a VP shunt.

\section{Disclosure}

Katsuyuki Hotta and Katsuyuki Kiura received research grants and honoraria from Chugai Pharmaceutical, Eli Lilly, and AstraZeneca. The other authors report no further conflicts of interest in this work.

\section{References}

1. Gleissner B, Chamberlain MC. Neoplastic meningitis. Lancet Neurol. 2006;5(5):443-452.

2. Lee SJ, Lee JI, Nam DH, et al. Leptomeningeal carcinomatosis in non-small-cell lung cancer patients: impact on survival and correlated prognostic factors. J Thorac Oncol. 2013;8(2):185-191.

3. Morris PG, Reiner AS, Szenberg OR, et al. Leptomeningeal metastasis from non-small cell lung cancer: survival and the impact of whole brain radiotherapy. $J$ Thorac Oncol. 2012;7(2):382-385.

4. Gwak HS, Joo J, Kim S, et al. Analysis of treatment outcomes of intraventricular chemotherapy in 105 patients for leptomeningeal carcinomatosis from non-small-cell lung cancer. J Thorac Oncol. 2013; 8(5):599-605.

5. Umemura S, Tsubouchi K, Yoshioka H, et al. Clinical outcome in patients with leptomeningeal metastasis from non-small cell lung cancer: Okayama Lung Cancer Study Group. Lung Cancer. 2012;77(1): 134-139.

6. Ichihara E, Hotta K, Nogami N, et al. Phase II trial of gefitinib in combination with bevacizumab as first-line therapy for advanced non-small cell lung cancer with activating EGFR gene mutations: the Okayama Lung Cancer Study Group Trial 1001. J Thorac Oncol. 2015; 10(3):486-491.

7. Seto T, Kato T, Nishio M, et al. Erlotinib alone or with bevacizumab as first-line therapy in patients with advanced non-squamous nonsmall-cell lung cancer harbouring EGFR mutations (JO25567): an open-label, randomised, multicentre, phase 2 study. Lancet Oncol. 2014; 15(11):1236-1244.

8. Lee E, Keam B, Kim DW, et al. Erlotinib versus gefitinib for control of leptomeningeal carcinomatosis in non-small-cell lung cancer. J Thorac Oncol. 2013;8(8):1069-1074.

9. Masuda T, Hattori N, Hamada A, et al. Erlotinib efficacy and cerebrospinal fluid concentration in patients with lung adenocarcinoma developing leptomeningeal metastases during gefitinib therapy. Cancer Chemother Pharmacol. 2011;67(6):1465-1469.

10. Yi HG, Kim HJ, Kim YJ, et al. Epidermal growth factor receptor (EGFR) tyrosine kinase inhibitors (TKIs) are effective for leptomeningeal metastasis from non-small cell lung cancer patients with sensitive EGFR mutation or other predictive factors of good response for EGFR TKI. Lung Cancer. 2009;65(1):80-84.

11. Jackman DM, Holmes AJ, Lindeman N, et al. Response and resistance in a non-small-cell lung cancer patient with an epidermal growth factor receptor mutation and leptomeningeal metastases treated with high-dose gefitinib. J Clin Oncol. 2006;24(27):4517-4520. 
12. Togashi Y, Masago K, Masuda S, et al. Cerebrospinal fluid concentration of gefitinib and erlotinib in patients with non-small cell lung cancer. Cancer Chemother Pharmacol. 2012;70(3):399-405.

13. Omuro AM, Lallana EC, Bilsky MH, DeAngelis LM. Ventriculoperitoneal shunt in patients with leptomeningeal metastasis. Neurology. 2005;64(9):1625-1627.

14. Lee SH, Kong DS, Seol HJ, Nam DH, Lee JI. Ventriculoperitoneal shunt for hydrocephalus caused by central nervous system metastasis. J Neurooncol. 2011;104(2):545-551.

15. Nagano T, Kotani Y, Kobayashi K, et al. Long-term outcome after multidisciplinary approach for leptomeningeal carcinomatosis in a non-small cell lung cancer patient with poor performance status. Intern Med. 2011;50(24):3019-3022.

16. So T, Inoue M, Chikaishi Y, Nose N, Sugio K, Yasumoto K. Gefitinib and a ventriculo-peritoneal shunt to manage carcinomatous meningitis from non-small-cell lung cancer: report of two cases. Surg Today. 2009; 39(7):598-602.

17. Kubo T, Takigawa N, Kiura K, et al. Efficacy of a lumbo-peritoneal shunt for meningeal carcinomatosis refractory to gefitinib treatment. Anticancer Res. 2009;29(7):2759-2760.

18. Herrlinger U, Wiendl H, Renninger M, Forschler H, Dichgans J, Weller M. Vascular endothelial growth factor (VEGF) in leptomeningeal metastasis: diagnostic and prognostic value. Br J Cancer. 2004; 91(2):219-224.

19. Groves MD, Hess KR, Puduvalli VK, et al. Biomarkers of disease: cerebrospinal fluid vascular endothelial growth factor (VEGF) and stromal cell derived factor (SDF)-1 levels in patients with neoplastic meningitis (NM) due to breast cancer, lung cancer and melanoma. J Neurooncol. 2009;94(2):229-234.
20. Besse B, Le Moulec S, Mazieres J, et al. Bevacizumab in patients with nonsquamous non-small cell lung cancer and asymptomatic, untreated brain metastases (BRAIN): a nonrandomized, phase II study. Clin Cancer Res. 2015;21(8):1896-1903.

21. Dickson PV, Hamner JB, Sims TL, et al. Bevacizumab-induced transient remodeling of the vasculature in neuroblastoma xenografts results in improved delivery and efficacy of systemically administered chemotherapy. Clin Cancer Res. 2007;13(13):3942-3950.

22. Wildiers H, Guetens G, De Boeck G, et al. Effect of antivascular endothelial growth factor treatment on the intratumoral uptake of CPT-11. Br J Cancer. 2003;88(12):1979-1986.

23. Chatterjee S, Wieczorek C, Schottle J, et al. Transient antiangiogenic treatment improves delivery of cytotoxic compounds and therapeutic outcome in lung cancer. Cancer Res. 2014;74(10):2816-2824.

24. Chinot OL, Wick W, Mason W, et al. Bevacizumab plus radiotherapytemozolomide for newly diagnosed glioblastoma. $N$ Engl J Med. 2014;370(8):709-722.

25. Levin VA, Bidaut L, Hou P, et al. Randomized double-blind placebocontrolled trial of bevacizumab therapy for radiation necrosis of the central nervous system. Int J Radiat Oncol Biol Phys. 2011;79(5): 1487-1495
OncoTargets and Therapy

\section{Publish your work in this journal}

OncoTargets and Therapy is an international, peer-reviewed, open access journal focusing on the pathological basis of all cancers, potential targets for therapy and treatment protocols employed to improve the management of cancer patients. The journal also focuses on the impact of management programs and new therapeutic agents and protocols on

\section{Dovepress}

patient perspectives such as quality of life, adherence and satisfaction The manuscript management system is completely online and includes a very quick and fair peer-review system, which is all easy to use. Visit http://www.dovepress.com/testimonials.php to read real quotes from published authors. 